\title{
Stimulation of NREM Delta EEG by Ketamine Administration during Waking: Demonstration of Dose Dependence
}

Last year we reported in this journal that three intraperitoneal injections of ketamine in rats during the waking (dark) period increased delta EEG during subsequent NREM sleep in the light period (Feinberg and Campbell 1993). Our data suggested these effects were dose dependent. However, because we had an insufficient number of animals to perform either a pure within-subjects or between-subjects analysis of variance, dose dependence was not statistically demonstrated. In this initial study we found no difference between the EEG effects of 15 and $25 \mathrm{mg} / \mathrm{Kg}$. To test for dose dependence between 25 and $50 \mathrm{mg} / \mathrm{Kg}$ of ketamine, we studied three additional rats with $25 \mathrm{mg} / \mathrm{Kg}$ of ketamine, enabling us to perform an analysis of variance with dose $(25 \mathrm{or} 50 \mathrm{mg} / \mathrm{Kg})$ as a grouping factor ( $n=5$ /group). Figure 1 shows significant dose dependence for each delta ( 1 to $4 \mathrm{~Hz}$ ) EEG measure.

Ketamine increases limbic system metabolism. The delta increase following its waking administration is, therefore, consistent with the "intensity" hypothesis of the homeostatic model of sleep (Feinberg 1974) that prompted our original study. Whatever its theoretical significance, the magnitude of delta stimulation shown in Figure 1 for $50 \mathrm{mg} / \mathrm{kg}$ ketamine appears to exceed that produced by any other drug. Our hypothesis that these effects are mediated by ketamine's blockade of the NMDA-gated cation channel is supported by recent studies in our laboratory that show equally robust delta stimulation by $\mathrm{MK}-801$, a more specific NMDA channel blocker (Campbell and Feinberg, in preparation).

In addition to their relevance for the homeostatic model of delta sleep, our findings could bear on basic and clinical issues, including the neurotransmitter regulation of sleep and the development of a new class of hypnotics. However, as we noted in our original report,

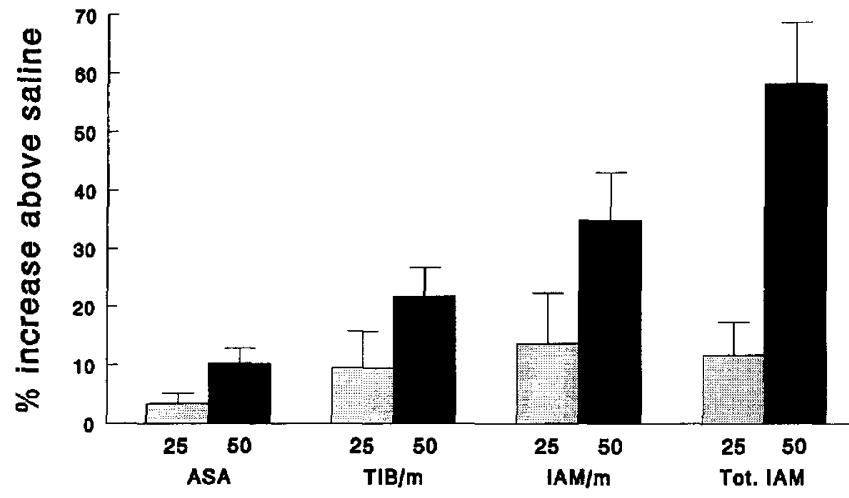

Figure 1. Mean (+SE) percentage increase above saline levels for delta ( 1 to $4 \mathrm{~Hz}$ ) EEG in NREM sleep during the 12 hour light (inactive) period in Sprague-Dawley rats. The animals had received three injections of $25 \mathrm{mg} / \mathrm{kg}$ or $50 \mathrm{mg} / \mathrm{kg}$ ketamine during the dark (active) period. $n=5$ in each dosage group. Dose effects were significant for each delta variable by ANOVA BMDP 2V, (df 1, 7): ASA, (average sample amplitude) $\mathrm{F}=7.4, p=.03 ; \mathrm{TIB} / \mathrm{m}$ (time in delta band $/ \mathrm{min}$ ) $\mathrm{F}=11.3, p=.01 ; \mathrm{IA} / \mathrm{m}$ (integrated amplitude $/ \mathrm{min}$ ) $\mathrm{F}=$ $8.5, p=.02$; Tot. IA/M (total integrated amplitude in light period) $\mathrm{F}=13.6, p=.008$

one cannot conclude that any increase in NREM delta EEG is, perforce, an enhancement of true (physiological) NREM sleep. Indeed, after publishing our findings, we became aware that ketamine, MK- 801 and similar noncompetitive NMDA cation channel blockers induce pathomorphological changes in rat brain (vacuolization in posterior cingulate and retrosplenial cortices) (Olney et al. 1989). These changes occur after equivalent dosages and show a postinjection time course that parallels the delta increase. For this reason, the delta increase following ketamine may not represent a true in- 
tensification of NREM sleep; instead, it may represent pathological slowing of the EEG caused by neurotoxicity. Such a pathological effect would itself be of interest in at least two respects. First, NREM delta EEG could be used to screen noninvasively for neurotoxic effects of NMDA cation channel blockers; such effects are of clinical concern because these drugs are currently being evaluated as treatments for the excitotoxic brain injury that follows ischemic stroke. Second, the ability to produce a massive increase in delta that occurs selectively in NREM sleep could provide a useful paradigm for studying the neurophysiology and pharmacology of delta wave generation. One last point is that the NREM delta effect could be closely correlated with neurotoxicity but still not be causally related; i.e., both could be independent consequences of some third factor, such as limbic system hypermetabolism). We have embarked on experiments to distinguish among these possibilities.

\section{ACKNOWLEDGMENTS}

This work was supported by research funds from the Department of Veterans Affairs.

I. Feinberg, M.D.

I.G. Campbell, M.S.

Department of Psychiatry

University of California-Davis

\section{REFERENCES}

Feinberg I (1974): Changes in sleep cycle patterns with age. J Psychiat Res 10:283-306

Feinberg I, Campbell IG (1993): Ketamine administration during waking increases delta EEG intensity in rat sleep. Neuropsychopharmacology 9:41-48

Olney JW, Labruyere J, Price MT (1989): Pathological changes induced in cerebrocortical neurons by phencyclidine and related drugs. Science 244:1360-1362 